\title{
ELECTROHYDRODYNAMIC DEFORMATION AND INTERACTION OF MICROSCALE DROP PAIRS
}

\author{
R. PILLAI ${ }^{1,2}$, J.D. BERRY ${ }^{1,3}$ D.J.E. HARVIE ${ }^{1,2}$ \& M.R. DAVIDSON ${ }^{1}$ \\ ${ }^{1}$ Department of Chemical and Biomolecular Engineering, The University of Melbourne, Australia. \\ ${ }^{2}$ Particulate Fluids Processing Centre (PFPC), The University of Melbourne, Australia. \\ ${ }^{3}$ CSIRO Mineral Resources, Australia.
}

\begin{abstract}
Binary drop electrocoalescence is the process of inducing two drops, suspended in an immiscible fluid, to coalesce in the presence of an external electric field. Electric forces have been known to accelerate the rupture of the interfacial film and enhance drop coalescence but the process has not been well characterized. The effects of the drop ion concentration and interfacial tension on the coalescence process are studied. It is shown that increasing interfacial tension, along with electric field makes it more likely that the drops stabilize after coalescence, as opposed to breaking up. This is due to the relative magnitudes of the drop deformation and charge separation timescales.
\end{abstract}

\section{INTRODUCTION}

In addition to its importance in industrial processes like emulsification and liquid-liquid extraction [1], drop-drop (or binary drop) coalescence phenomena have recently found application in microfluidic lab-on-a-chip (LOC) devices [2]. Binary drop electrocoalescence is the process of enhancement of the coalescence process between two drops by using an electric field. In particular, the electric forces aid the drainage and rupture of the interfacial film separating the drops in the region of contact [3]. The existing numerical studies on the topic employ electric field models that ignore the presence of ions inside the drops [4].

In this paper, the authors employ a recently developed model that allows for conductive, diffusive and advective fluxes of ions, and for diffuse regions of charge to form [5]. This is appropriate for modelling microfluidic drops, where electrokinetics play an important role [6-9]. Two identical microscale drops of electrolyte solution are suspended in oil and subjected to an external electric field. This system is commonly employed in microfluidic LOC devices and is numerically investigated here. Depending on the application, either complete coalescence, where the drops coalesce into a single larger drop, or partial coalescence, where part of the drop phase pinches off from the rest of the drop, is desirable [10]. These phenomena have not been well characterized and are studied in this paper.

\section{MODEL FORMULATION}

The system studied in this work is illustrated in Fig. 1. Two axisymmetric drops are considered, with permittivity $\varepsilon_{\mathrm{d}}$ (subscripts $d$ and $c$ are used to denote disperse and continuous phase properties, respectively). The drops contain symmetric anions and cations with number densities $n_{+}$and $n_{-}$, respectively, valencies $z_{+}=-z_{-}=z$, and diffusivities $\alpha_{+}=\alpha_{-}=\alpha$. The initial drop ion concentration, given by the geometric mean of the species ion concentrations $\left(n_{0}=\sqrt{n_{+} n_{-}}\right)$, is uniform

This paper is part of the Proceedings of the $8^{\text {th }}$ International Conference on Computational and Experimental Methods in Multiphase and Complex Flow (Multiphase Flow 2015)

www.witconferences.com
} 


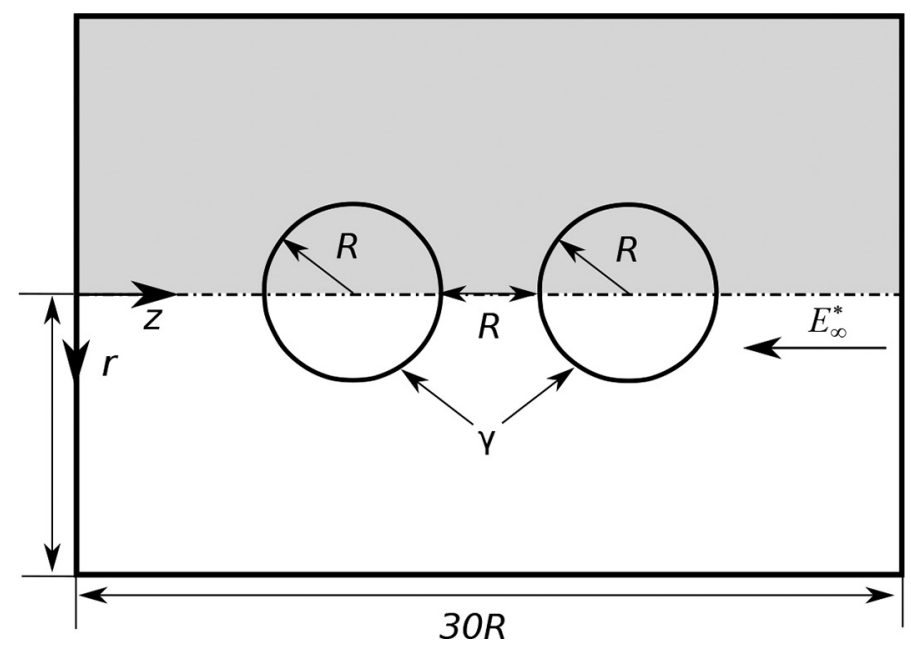

Figure 1: Schematic of an axisymmetric, binary electrolytic drop system (symmetry boundary conditions applied on the horizontal centreline). Both the drops have radius $R$ and are initially separated by a distance $R$. They are suspended in an immiscible fluid, where $\gamma$ is the interfacial tension, and are acted on by an external electric field $\left(E_{\infty}^{*}\right)$ in the $z$ direction.

everywhere. The drops are suspended in a dielectric medium, oil, of permittivity $\varepsilon_{\mathrm{c}}$. The permittivity of the drops is greater than the oil $\left(\varepsilon_{\mathrm{d}} / \varepsilon_{\mathrm{c}}=50\right)$. To simplify the problem, the drops and the continuous phase are assumed to have equal viscosity $\left(\mu_{c}=\mu_{d}\right)$ and density $\left(\rho_{c}=\rho_{d}\right)$. These are reasonable assumptions as density has been shown to have negligible effect on drop deformation, and oils with viscosity close to that of water can be used in microfluidic devices [7].

The domain is a horizontally aligned, parallel plate capacitor with a separation distance of $30 R$ and width $4 R$. The drops are assumed to be spherical initially with radii $R$ and are located at the centre of the domain with an initial horizontal separation distance of $R$. As the drops are electrically neutral, the axial electric field is set up to be equal at the left and right boundaries to satisfy Gauss' law in the domain $\left(E_{\infty}^{*}\right)$. The top and bottom boundaries have zero normal field conditions specified. This corresponds to a uniform external field $\boldsymbol{E}$ along the $z$ axial direction at large distances from the drops. The drops are assumed to be symmetrical along the horizontal centereline (as shown in figure Fig. 1), and the calculations are performed on the symmetric half domain. The interface between the drops and oil is assumed to have an interfacial tension $\gamma$.

Under the action of the electric field, the ions conduct towards the drop interfaces, forming a concentration gradient which is opposed by diffusion, resulting in the formation of a diffuse charge layer. In electrokinetics, the inverse dimensionless Debye length $\left(K_{\mathrm{d}}\right)$ is a measure of the total electrostatic effect of the mobile ions. For a fixed geometry and physical parameters, an increase in $K_{\mathrm{d}}$ here translates to an increase in the number density of ions inside the drop $\left(n_{0}\right)$. In this paper, $K_{\mathrm{d}}$ is considered as a dimensionless ion concentration.

\subsection{Governing equations}

The equations governing the system are non-dimensionalized using a characteristic length scale $R$, permittivity scale $\varepsilon_{\mathrm{d}}$, ion scale $n_{0}$, velocity scale $V_{\text {ref }}$ and an electric field scale $E_{\text {ref }}$, where $V_{\text {ref }}$ and $E_{\text {ref }}$ will be specified later: 


$$
x=\frac{x^{*}}{R} ; \boldsymbol{u}=\frac{\boldsymbol{v}^{*}}{V_{\mathrm{ref}}} ; \mu=\frac{\mu^{*}}{\mu_{\mathrm{d}}} ; \rho=\frac{\rho^{*}}{\rho_{\mathrm{d}}} ; \varepsilon=\frac{\varepsilon^{*}}{\varepsilon_{\mathrm{d}}} ; n_{ \pm}=\frac{n_{ \pm}^{*}}{n_{0}} ; \boldsymbol{E}=\frac{\boldsymbol{E}^{*}}{E_{\mathrm{ref}}}
$$

where '*' denotes a physical variable, $\boldsymbol{u}$ is the fluid velocity vector and $\boldsymbol{E}^{*}$ is the dimensional electric field. This gives rise to the dimensionless groups:

$$
\mathrm{Re}=\frac{\rho_{\mathrm{d}} V R}{\mu_{\mathrm{d}}} ; \mathrm{We}=\frac{\rho_{\mathrm{d}} V^{2} R}{\gamma} ; \mathrm{Pe}=\frac{V R}{\alpha} ; \mathrm{B}=\frac{\rho_{\mathrm{d}} k^{2} T^{2} \varepsilon_{0} \varepsilon_{\mathrm{d}}}{2 z^{2} e^{2} \mu_{\mathrm{d}}^{2}} ; K_{\mathrm{d}}=\sqrt{\frac{2 z^{2} e^{2} n_{0} R^{2}}{\varepsilon_{0} \varepsilon_{\mathrm{d}} k T}}
$$

where Re is the Reynolds number, We the Weber number, Pe the Péclet number, B is a parameter that depends on the temperature of water, and $K_{\mathrm{d}}$ is the dimensionless inverse Debye length mentioned previously. $e$ and $k$ are the electron charge and Boltzmann constant, respectively. The dimensionless governing equations are

$$
\begin{gathered}
\nabla \cdot \boldsymbol{u}=0 \\
\frac{\partial \rho \boldsymbol{u}}{\partial t}+\nabla \cdot(\rho \boldsymbol{u} \boldsymbol{u})=-\nabla p+\frac{1}{\mathrm{Re}} \nabla \cdot t_{\boldsymbol{V}}+\frac{1}{\mathrm{We}} \boldsymbol{F}_{\mathrm{S}}+\frac{2 \mathrm{~B}}{\mathrm{Re}^{2}} \boldsymbol{F}_{\mathrm{E}} \\
\frac{\partial c n_{ \pm}}{\partial t}+\nabla \cdot\left(\boldsymbol{u} c n_{ \pm}\right)=\frac{1}{\mathrm{Pe}} \nabla \cdot\left(c \nabla n_{ \pm} \mp c n_{ \pm} \boldsymbol{E}\right) \\
\nabla \cdot(\varepsilon \boldsymbol{E})=\frac{1}{2} K_{\mathrm{d}}^{2}\left(n_{+}-n_{-}\right) \\
\frac{\partial c}{\partial t}+\nabla \cdot(c \boldsymbol{u})=0
\end{gathered}
$$

Equation (5) is the transport equation for the disperse phase volume fraction, while $p$ denotes pressure, $\boldsymbol{\tau}_{\boldsymbol{V}}$ is the viscous stress tensor, $c$ is the VOF fractional volume function of the drop phase and $\boldsymbol{F}_{\mathbf{S}}$ is the force due to the interfacial tension. $\boldsymbol{F}_{\mathbf{E}}$ is the electrical force term represented by the divergence of the Maxwell stress tensor and $n_{+}-n_{-}$is the dimensionless charge density. Equations (1)-(5) are solved using the algorithm of Berry et al. [5] on a staggered, uniform mesh.

\subsection{Problem setup}

A thermal electric field scale of $E_{\mathrm{ref}}=k T / z e R$ is selected. The velocity scale is based on the capillary thinning velocity $\left(R / t_{\text {visc }}\right)$, where $t_{\text {visc }}$ is the viscocapillary time scale $\left(t_{\text {visc }}=\mu_{\mathrm{d}} R / \gamma\right)$. This gives $V_{\text {ref }}=\gamma / \mu_{\mathrm{d}}$, which means that the viscous capillary number, $\mathrm{Ca}=1$. Based on the scaling employed, the square of the Ohnesorge number $\left(\mathrm{Oh}^{2}=\mathrm{We} / \mathrm{Re}^{2}=\mu_{\mathrm{d}}^{2} / \rho_{\mathrm{d}} R_{\mathrm{d}} \gamma\right)$, a dimensionless number that depends on the experimental setup, is used to characterize the interfacial tension of the system. For the purposes of this study, the interfacial tension is varied from $10^{0}$ to $10^{-3} \mathrm{mN} / \mathrm{m}$, typical of water-oil interfaces with varying amounts of surfactant [11]. For drops with radius $100 \mu \mathrm{m}$ (a typical dimension for microfluidic systems), viscosity $10^{-3} \mathrm{~Pa} . \mathrm{s}$ 
and density $10^{3} \mathrm{~kg} / \mathrm{m}^{3}$ (typical values for aqueous drops), a corresponding range for $\mathrm{Oh}^{2}$ is obtained $\left(10^{1} \geq \mathrm{Oh}^{2} \geq 10^{-2}\right.$ as $\left.10^{-3} \leq \gamma \leq 10^{0} \mathrm{mN} / \mathrm{m}\right)$.

The two primary forces involved in binary drop coalescence are the electric forces deforming the drops and the interfacial tension forces resisting deformation (dependent on $\boldsymbol{E}$ and $\gamma$, respectively). Selecting an appropriate value of external electric field for binary drop systems with varying interfacial tension, however, is not straightforward. If a moderate value of electric field is chosen, such that the drops coalesce for the middle value of interfacial tension used $\left(\mathrm{Oh}^{2}=0.5\right)$, then the deformation behaviour of the drops changes drastically along the range of $\mathrm{Oh}^{2}$, from deforming negligibly (at the lowest $\mathrm{Oh}^{2} /$ highest $\gamma$ ) to disintegrating almost instantly (at the highest $\mathrm{Oh}^{2} /$ lowest $\gamma$ ). Either of these outcomes has little practical application when choosing parameters for designing microfluidic devices. It is instead desirable to study binary drop-oil coalescence behaviour where the electric field is sufficiently high for coalescence to occur, but low enough to ensure sufficient control over drop behaviour, for different combinations of water-oil interfacial tension. Therefore, instead of using a constant electric field, a constant ratio of electric forces to interfacial tension forces is used here, represented by the dimensionless electric capillary number $\left(C a_{\mathrm{E}}=\varepsilon_{0} \varepsilon_{\mathrm{c}} R E_{\propto}^{* 2} / \gamma\right.$. This number is fixed $\left(C a_{\mathrm{E}}=0.25\right)$ for all the cases considered in this paper. In effect, the external dimensional electric field at large distances from the drops $\left(E_{\infty}^{*}\right)$ increases from $\sim 6 \mathrm{kV} / \mathrm{m}$ to $\sim 6000 \mathrm{kV} / \mathrm{m}$ as $\mathrm{Oh}^{2}$ decreases from $10^{1}$ to $10^{-2}$.

\section{RESULTS}

\subsection{Permittivity force, charge force and single drop breakup}

Equation (3) shows that the electric field conducts the initially uniformly distributed ion species in opposite directions inside the drops. As a consequence, regions of depletion of one ion species relative to the other (i.e. charge) are formed. Hence, there are two electric forces deforming the drops. The first is the normal electric force formed at the interface due to the jump in permittivities from the drop phase to the continuous phase, and the second is the force due to the formation of free charge in the drop which is acted upon by the field. These are referred to as the 'permittivity force' and 'charge force', respectively.

Prior to studying binary drop coalescence, the simpler problem of single drop deformation, which was studied by the authors previously [7-9], is considered in Fig. 2. The contours of charge density magnitude are overlaid inside the drop. The horizontal field conducts the ion species, forming symmetric regions of charge separation at the left and right ends of the drop in the second frame. The combination of the two electric forces overcomes the opposing interfacial tension and deforms the drop in the third, fourth and fifth frames. This process continues until the drop breaks up, ejecting droplets from both ends in the last frame.

\subsection{Binary drop coalescence}

The results for binary drop coalescence for $\mathrm{Oh}^{2}=10$ and $K_{\mathrm{d}}=5$ are shown in Fig. 3. The contours of charge separation are overlaid, as was the case with Fig. 2. In fact, the charge separation process is remarkably similar to that shown in Fig. 2 particularly in the bottom three frames. Both (single and binary drop) cases show charge accumulation at the ends, followed by necking and pinching off of ejected droplets. The key difference (in addition to the larger size of the ejected droplets) is that the body of the drop in Fig. 2, prior to ejection 


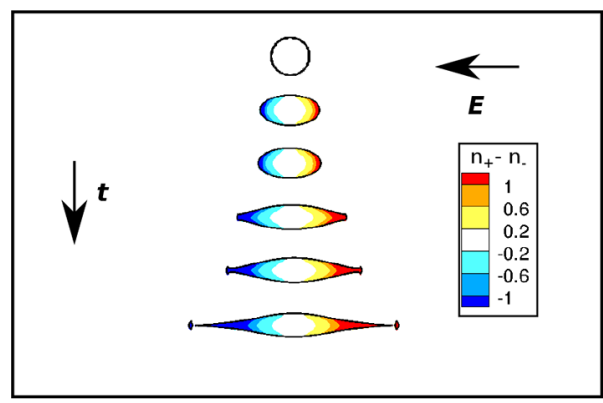

Figure 2: Single drop deformation and breakup for $\mathrm{Oh}^{2}=10$ and $K_{\mathrm{d}}=5$, with overlaid charge contours (in greyscale, the colour map can be interpreted as charge magnitude). The electric field acts horizontally and snapshots at different times are arranged vertically from initial state (top) to breakup (bottom). The dimensionless times for the individual frames are $t=0,5.5,10,25.5,27.5$ and 32 .

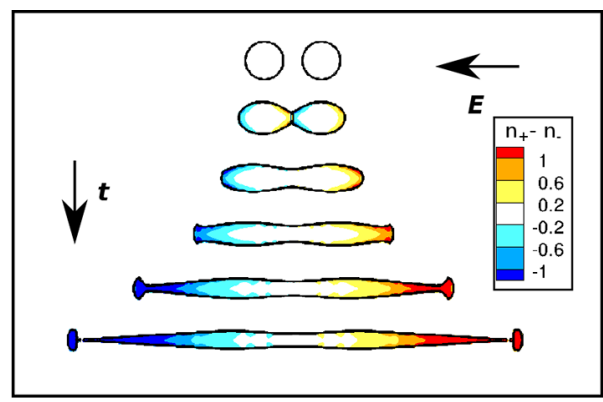

Figure 3: Binary drop coalescence for $\mathrm{Oh}^{2}=10$ and $K_{\mathrm{d}}=5$, with overlaid charge contours (in greyscale, the colour map can be interpreted as charge magnitude). The electric field acts horizontally and snapshots at different times are arranged vertically from initial state (top) to breakup (bottom). The dimensionless times for the individual frames are $t=0,5.5,10,14,20$ and 26.5 .

of droplets, is single lobed. In contrast, the body of the drop in Fig. 3 is two-lobed, with a narrower region in the centre. This is because this case starts off as two separate drops as seen in the top frame. Due to the application of the horizontal electric field, both drops develop regions of charge. The effect of the consequent charge force, in conjunction with the permittivity force, deforms both drops until they come in contact with each other, as shown in the second frame. At this point, in the absence of an electric field, the interfacial film of oil between the drops would take a finite amount of time to drain. Once the drainage had completed, the film would rupture and the drops would undergo a topological transition to a single, unified interface. Then, the interfacial tension forces would act to minimize the total surface area of the conjoined drops, resulting in the formation of a larger spherical drop over time. In the case studied here, however, there is an electric field, and the electric forces accelerate the rupture of the interfacial film, which occurs almost instantaneously [12]. Once the drops coalesce, the regions of charge that existed on either side of the drop-drop interface before it ruptured are neutralized by each other, as they have opposite signs. However, there 


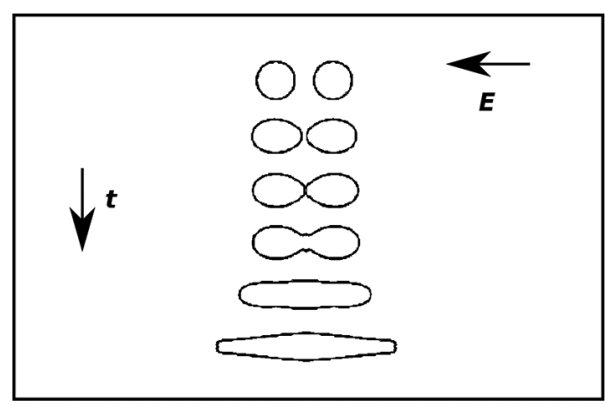

Figure 4: Binary drop coalescence for $\mathrm{Oh}^{2}=10^{-2}$ and $K_{\mathrm{d}}=5$. The electric field acts horizontally and snapshots at different times are arranged vertically from initial state (top) to completion of coalescence (bottom). The dimensionless times for the individual frames are $t=0,0.12,0.13,0.14,0.27$ and 0.48 .

are still regions of charge at the two other ends of the original drops, which now form the two ends of the longer unified structure. The electric forces acting at the ends of the unified drop cannot be balanced by the interfacial tension, and the drop therefore does not assume a spherical shape. Instead, the ends continue to extend until necking, and consequent pinching off of droplets, is achieved.

\subsection{Effect of interfacial tension}

Figure 4 illustrates the coalescence of a binary drop system with the same drop ion concentration as in Fig. $3\left(K_{\mathrm{d}}=5\right)$, but higher interfacial tension $\left(\mathrm{Oh}^{2}=10^{-2}\right)$. The first three frames show a similar process of electric forces deforming the interfaces and bringing them in contact. In the second frame, the inner tips of the drops have a more pronounced curvature than the outer tips. This indicates that the oppositely charged regions on either side of the approaching drop ends exert an electrostatic attraction on each other. However, more significantly after coalescing, the coalesced drop does not breakup like the preceding case as shown in Fig. 3. Instead, it elongates to form a single-lobed ellipsoid with flat ends. Given that the charge force, which predominates drop breakup, depends on $K_{\mathrm{d}}$, which is the same for both cases; it is not immediately clear why the higher interfacial tension results in different drop dynamics given that the ratio between applied electric field and interfacial tension remains constant using our chosen scalings. The reason the drops behave differently is due to the relative timescale for drop deformation compared with that of charge separation, quantified by the Péclet number (Pe). The Pe for the case in Fig. 3 is 10 and increases to $10^{4}$ for the case in Fig. 4, implying that charge separation is less important for the latter. The constant $C a_{\mathrm{E}}$ ensures that the ratio of electric forces and interfacial tension forces is roughly similar for Figs 3 and 4. However, for the lower $\mathrm{Oh}^{2}$ (and higher electric field) case, the composition of the electric force changes [9]. The quicker drop deformation prevents significant charge separation from occurring and prevents the charge force from playing an important role. Instead, the permittivity force, which varies as the square of the electric field, contributes a larger percentage of the electric force. This is because, unlike the charge force, the permittivity force depends only on the external electric field and the permittivity jump at the interface and acts instantaneously. However, as the permittivity force acts normal to the drop interface (as opposed to the charge forces which can 


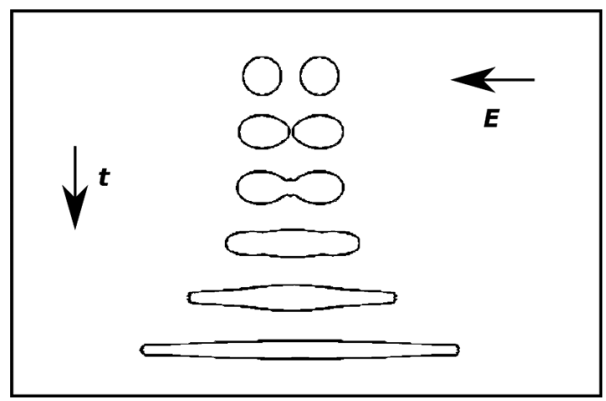

Figure 5: Binary drop coalescence for $\mathrm{Oh}^{2}=10^{-2}$ and $K_{\mathrm{d}}=25$. The electric field acts horizontally and snapshots at different times are arranged vertically from initial state (top) to completion of coalescence (bottom). The dimensionless times for the individual frames are $t=0,0.12,0.14,0.27,0.54$ and 1.12 .

create tangential stresses), it cannot destabilize the drop tips. Hence, the drop does not break up for the time observed.

\subsection{Effect of drop ion concentration}

In Fig. 5, the binary drop coalescence process for a system with the same $\mathrm{Oh}^{2}$ as Fig. 4 is illustrated, but for a higher drop ion concentration $\left(K_{\mathrm{d}}=25\right)$. The first four frames look identical to the first, third, fourth and fifth frames of Fig. 4. This is not surprising as the permittivity force, which dominates the initial drop deformation, is similar in both cases as the permittivities of the drops and the external electric field are the same. However, the fifth frame shows the coalesced drop extending further than the case shown in Fig. 4. The drop continues to extend for a much longer period of time, finally assuming the elongated shape in the last frame. The greater number density of the ions in the drop results in a greater charge force. While this does not affect the coalescence process, which in this case depends more on permittivity force, the charge separation continues post coalescence and extends the drop further than was the case in Fig. 4. The drop is still stable (for the time observed), implying that the charge force is still insufficient to initiate drop breakup.

Figure 6 illustrates with the same $K_{\mathrm{d}}$ as Fig. 5, but a higher $\mathrm{Oh}^{2}$ of $10^{-1}$, which translates to a lower field. This results in a lower permittivity force and a delayed coalescence, compared to the case in Fig. 5. This allows the ions to separate to a greater extent, and the resultant charge separation forms the familiar pattern of lobe formation, followed by ejection of droplets, that was seen in Fig. 3. However, when compared to Fig. 3 which had $\mathrm{Oh}^{2}=10^{1}$ and therefore deformed slower, a higher $K_{\mathrm{d}}$ is required to break up the unified drop in Fig. 6 . This is because the larger initial number density of ions compensates for the fact that a smaller proportion of the ions, as a percentage, is separated in the quicker deformation process. Note that, unlike the case in Fig. 3 where a two-lobed drop (i.e. thinner at the coalescence midplane) was formed, the unified drop here is single lobed, because the drops coalesce completely (as seen in frame 3 ) prior to breakup.

\section{CONCLUSIONS}

Simulations are presented for the coalescence of binary aqueous drops suspended in oil, acted on by an external electric field. The effects of interfacial tension and drop ion concentration 


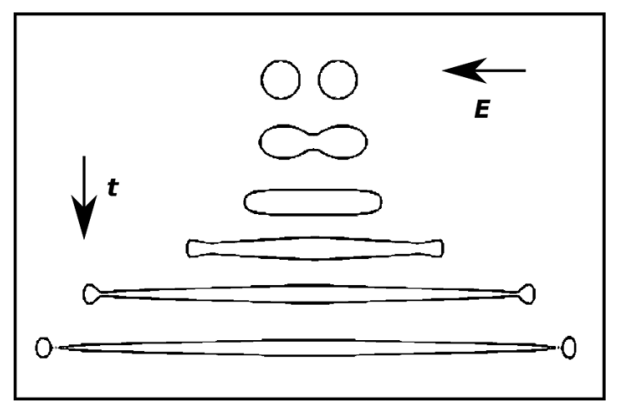

Figure 6: Binary drop coalescence for $\mathrm{Oh}^{2}=10^{-1}$ and $K_{\mathrm{d}}=25$. The electric field acts horizontally and snapshots at different times are arranged vertically from initial state (top) to completion of coalescence (bottom). The dimensionless times for the individual frames are $t=0,0.70,1.45,2.80,4.45$ and 5.20.

are studied, for a fixed electric capillary number $\left(C a_{\mathrm{E}}=0.25\right)$. It is shown that the higher the interfacial tension (and the electric field), the higher the importance of permittivity force on binary drop coalescence as differences in drop ion concentration do not then significantly affect the coalescence behaviour. For the higher value of interfacial tension considered here, the drops coalesce and then elongate to a flat-ended ellipsoid. The faster drop coalescence and elongation prevent the charge force from playing a significant role, and the coalesced drop does not break up during the simulation time.

For the lower value of interfacial tension considered (and lower electric field), the timescale of drop deformation is slow enough for charge separation, and hence the charge force, to play a significant role. Consequently, the charge accumulating at the ends produces sufficient electrical force to destabilize the drop tips, and droplets are ejected from both ends. For the intermediate value of interfacial tension considered, a higher drop ion concentration was required to achieve breakup. This was because the faster deformation process was compensated by the higher ion density in the drops.

Depending on the application, either partial or complete binary drop coalescence is desirable [10]. Our results show that partial coalescence becomes more likely as the interfacial tension of the system is decreased, for a fixed interfacial tension to electric field ratio (represented by $C a_{\mathrm{E}}=0.25$ ). For fixed interfacial tension, partial coalescence becomes more likely as drop ion concentration is increased. This is because accumulation of charge at the ends of the coalesced drop, which plays a key role in drop breakup, is more likely to occur under these conditions.

\section{ACKNOWLEDGEMENTS}

R. Pillai acknowledges the support of a Melbourne International Research Scholarship (MIRS) during the completion of this work, and a PFPC travel grant to attend the conference.

\section{REFERENCES}

[1] Eiswirth, R.T., Bart, H.J., Ganguli, A.A. \& Kenig, E.Y., Experimental and numerical investigation of binary coalescence: liquid bridge building and internal flow fields. Physics of Fluids, 24(6), p. 062108, 2012. doi: http://dx.doi.org/10.1063/1.4729791

[2] Curran, K., Colin, S., Baldas, L. \& Davies, M., Liquid bridge instability applied to microfluidics. Microfluidics and Nanofluidics, 1, pp. 336-345, 2005. doi: http://dx.doi. org/10.1007/s10404-005-0038-7 
[3] Eow, J., Ghadiri, M., Sharif, A. \& Williams, T., Electrostatic enhancement of coalescence of water droplets in oil: a review of the current understanding. Chemical Engineering Journal, 84(3), pp. 173-192, 2001. doi: http://dx.doi.org/10.1016/S13858947(00)00386-7

[4] Atten, P., Lundgaard, L. \& Berg, G., A simplified model of electrocoalescence of two close water droplets in oil. Journal of Electrostatics, 64(7-9), pp. 550-554, 2006. doi: http://dx.doi.org/10.1016/j.elstat.2005.10.009

[5] Berry, J.D., Davidson, M.R. \& Harvie, D.J.E., A multiphase electrokinetic flow model for electrolytes with liquid/liquid interfaces. Journal of Computational Physics, 251, pp. 209-222, 2013. doi: http://dx.doi.org/10.1016/j.jcp.2013.05.026

[6] Squires, T. \& Quake, S., Microfluidics: fluid physics at the nanoliter scale. Reviews of Modern Physics, 77, pp. 977-1026, 2005. doi: http://dx.doi.org/10.1103/RevModPhys.77.977

[7] Pillai, R., Berry, J.D., Harvie, D.J.E. \& Davidson, M.R., Effect of interfacial tension and electric field on charge separation dynamics inside stable and unstable microdrops, 19th Australasian Fluid Mechanics Conference, Melbourne, Australia, December 8-11, 2014.

[8] Pillai, R., Berry, J.D., Harvie, D.J.E. \& Davidson, M.R. , Electrolytic drops in an electric field: a numerical study of drop deformation and breakup. Physical Review E, 92, 013007, pp. 1-15, 2015. doi: http://dx.doi.org/10.1103/physreve.92.013007

[9] Pillai, R., Berry, J.D., Davidson, M.R. \& Harvie, D.J.E., Electrokinetics of isolated electrified drops. 2015 (submitted to Soft Matter).

[10] Minardi, C.S., Taghioskoui, M., Jang, S.J. \& Jorabchi, K., Reagent delivery by partial coalescence and noncoalescence of aqueous microdroplets in oil. Analytical chemistry, 85(13), pp. 6491-6496, 2013. doi: http://dx.doi.org/10.1021/ac4010524

[11] Xu, J., Zhang, Y., Chen, H., Wang, P., Xie, Z., Yao, Y., Yan, Y. \& Zhang, J., Effect of surfactant headgroups on the oil/water interface: an interfacial tension measurement and simulation study. Journal of Molecular Structure, 1052, pp. 50-56, 2013. doi: http://dx.doi.org/10.1016/j.molstruc.2013.07.049

[12] Aryafar, H. \& Kavehpour, H.P., Electrocoalescence: effects of DC electric fields on coalescence of drops at planar interfaces. Langmuir: The ACS Journal of Surfaces and Colloids, 25(21), pp. 12460-12465, 2009. doi: http://dx.doi.org/10.1021/la902758u 